WellBeing International

WBI Studies Repository

9-1999

\title{
Partial Tooth-Clipping of Suckling Pigs: Effects on Neonatal Competition and Facial Injuries
}

\author{
Daniel M. Weary \\ University of British Columbia \\ David Fraser \\ University of British Columbia
}

Follow this and additional works at: https://www.wellbeingintlstudiesrepository.org/farahus

Part of the Agribusiness Commons, Animal Studies Commons, and the Operations and Supply Chain Management Commons

Recommended Citation

FINAL EDITED VERSION OF MATERIAL. NOT FORMATTED FOR PUBLICATION.

This material is brought to you for free and open access by WellBeing International. It has been accepted for inclusion by an authorized administrator of the WBI Studies Repository. For more information, please contact wbisr-info@wellbeingintl.org.

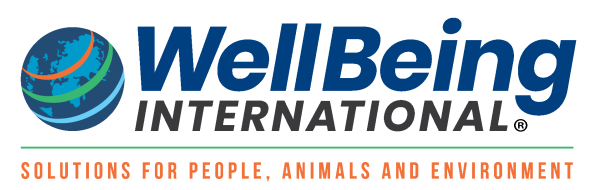




\title{
Partial Tooth-Clipping of Suckling Pigs: Effects on Neonatal Competition and Facial Injuries
}

\author{
Daniel M. Weary and David Fraser \\ University of British Columbia
}

\author{
KEYWORDS \\ body weight, social behavior, dentition, mortality, piglet, teeth
}

\begin{abstract}
Piglets are born with fully erupted 'needle teeth' which are used in competition for access to teats. Producers often clip these teeth to the gum line soon after birth to avoid any resulting facial lacerations, but this clipping can itself lead to injury or infection. Two experiments investigated the effects on facial injury and piglet performance of the potentially less damaging practice of 'partial clipping' whereby only the distal third of the tooth is removed. In Experiment 1, teeth were (1) partially clipped, (2) fully clipped to the gum line, or (3) left intact. All piglets of a litter received one treatment on one side of the mouth and a different treatment on the other side, with the three treatments balanced over the two sides in different litters. Facial lesions, scored separately for the two sides of the face at 3,7 , and 14 days, were negligible on the side facing either partially clipped or fully clipped teeth, but lesions on the side facing intact teeth were significantly greater $(P<0.02)$. In Experiment 2, some piglets in each of 22 litters had their teeth partially clipped, fully clipped, or left intact. In the first week after birth, piglets with fully clipped teeth gained the least (132 $\pm 6 \mathrm{~g} /$ day), those with partially clipped teeth were intermediate $(144 \pm 7)$, and those with intact teeth gained the most $(154 \pm 7 ; P<0.02)$. There was no significant effect of treatment on later weight gain, the number of piglet deaths, or piglet suckling position (anterior to posterior). Thus, teeth that were partially clipped appeared to have a slight effect in competition among litter-mates, but caused little injury to other piglets.
\end{abstract}

\section{Introduction}

Piglets are born with eight fully erupted 'needle teeth' (the deciduous canines and third incisors), which the animals use to deliver sideward bites to the faces of litter-mates when fighting at the udder. Producers often clip these teeth to the gum line soon after birth. Clipping the teeth helps reduce facial injuries to piglets and is thought to reduce damage to the sow's udder. However, clipping can also reduce a piglet's ability to compete with litter-mates during teat disputes (Fraser and Thompson, 1991); it exposes the pulp cavity and allows for pulpitis and gingivitis; and it can damage the gums and cause splintering of the teeth, especially for teeth clipped close to the gum line (Burger, 1983; Hutter et al., 1994). 
Producers have at least three alternatives to clipping teeth to the gum line. One is to leave the teeth intact. Two recent studies involving piglets with intact teeth found little if any observable damage to the sow's udder (Robert et al., 1995; Brown et al., 1996), but piglets did receive facial injuries. However, Burger (1983) suggested that the direct and indirect damage caused by clipping may actually exceed the effects of injuries caused by leaving teeth intact. A second option is to remove the tips of the teeth with a special grinding tool. According to Hutter et al. (1994), this technique achieves the positive effects associated with clipping (i.e., fewer facial and udder injuries), while reducing the severity of drawbacks (i.e., damage to the tooth and gums). A third option is to clip only the tip of the tooth. Although this method is sometimes suggested in producer-oriented publications (e.g., Connor, 1993, p. 22), we are aware of no previous research that has evaluated its effectiveness.

The aim of the current study was to compare the effects of clipping the tips of the teeth vs. both complete clipping at the gum line and leaving the teeth intact. The comparison included effects on facial injuries (Experiment 1) and on piglet weight gains and competition for teats (Experiment 2).

\section{Animals, materials and methods}

\subsection{Experiment 1}

Experiment 1 involved 312 piglets from 28 litters with 10 or more piglets (mean \pm S.D. litter size $=11.5 \pm$ 1.3), all from a minimum-disease Landrace $\times$ Yorkshire research herd. Teeth were either intact (not clipped), partially clipped (clipped to remove an estimated one-third of the visible length of the tooth), or fully clipped (clipped to remove all of the tooth above the gum line). All clipping was performed by the same trained technician using clean, sharp side-cutting pliers, and was completed within $24 \mathrm{~h}$ of birth. Following standard practice for this herd, piglets were also ear-notched and received iron injections at this time.

Because previous work has shown large differences between litters in the severity of facial damage from intact teeth (Fraser, 1975), the experiment relied on within-litter comparisons. This was achieved by applying one clipping treatment to the right side of the mouth and another to the left side of the mouth for all piglets within a litter. When piglets compete for access to teats, they do so by delivering sideways bites, while facing the udder. Moreover, piglets normally remain on the same teat or teat pair throughout lactation (Fraser, 1975). Thus in this experiment, piglets competing at the udder faced teeth on one clipping treatment to their left and a different treatment to their right. Litters were assigned to one of three combinations: fully clipped on one side and intact on the other (10 litters), partially clipped on one side and intact on the other (9 litters), or fully clipped on one side and partially clipped on the other (9 litters). The clipping treatments were assigned to the left or right side in a counterbalanced manner, and were balanced across litter sizes. All piglets in a litter were clipped in the same manner.

An observer scored facial injuries on each side of each piglet's face. Injuries were scored when piglets were 3, 7, and 14 days of age using a four-point scale following Fraser (1975), running from 0 (no lesions visible) to 3 (severe lacerations with swelling, prominent wounds or dark scabs). Scores of 1 and 2 represented intermediate degrees of severity judged subjectively. To compare the effect of the clipping treatments, both sides of each piglet's face were scored, the difference between the scores was calculated and these differences were averaged for the litter. Mean differences ( $n=9$ or 10 litters) were then compared with the null expectation of 0 using a $t$-test. Mean values reported in Fig. 1 were calculated in the same way. In cases where any piglet in a litter received a score of 2 or more, all teeth were fully clipped to prevent further damage, and the litter was removed from further study. To avoid biasing the results because of litters that were removed, the largest score taken from each piglet was used in calculating the litter means. 


\subsection{Experiment 2}

Experiment 2 compared the effects of the three clipping treatments on piglets' weight gains and suckling position. The experiment involved 241 piglets from 22 litters, all from same research herd used in Experiment 1. Litters were selected with a minimum of nine healthy piglets (mean \pm S.D. litter size $=10.4$ \pm 0.5 ). Piglets were weighed on the first morning after birth. In each litter, the three heaviest piglets were treated as block 1, the next three heaviest as block 2, and the next three as block 3 . Within each block, one piglet was assigned at random to have its teeth clipped, another to be partially clipped, and the third remained intact. The technical procedure used for teeth clipping was the same as in Experiment 1. Clipping was again performed within $24 \mathrm{~h}$ of birth. Following standard practice for this herd, all piglets were ear-notched and received iron injections at the same time.

Piglets were weighed at the time of clipping (day 0), and weekly thereafter on days 7, 14, and 21. Between the ages of 14 and 21 days, the teat pair typically used by each piglet was determined using the procedure of Fraser and Thompson (1991). For each nursing episode, the piglets were removed to a holding box, marked on their backs with ink numbers for easy identification, and returned to the sow after a 50-min separation. During the nursings that ensued, an observer recorded which teat pair (1-7 anterior to posterior) was used by each piglet at the time of milk ejection. This procedure was repeated five times, and the observer recorded the teat pair used most commonly by each piglet.

Differences in teat number, birth weight and weight gain during each week (i.e., 1, 2, and 3) were analyzed separately by least squares analysis of variance. The general linear model included a term for litter (21 df ), so that the treatment effect was tested within litters. Because the three treatments involved different degrees of clipping, the treatment effect was tested with a linear term ( $1 \mathrm{df}$ ) plus a second term for the nonlinear component ( $1 \mathrm{df}$ ); both were tested against the residual error. In preliminary analyses, we tested for the effect of block (heaviest, middle, and lightest within each litter) and the interactions of treatment with litter size and treatment with sow parity, but these were never found to be significant. Only piglets that survived the duration of the experiment were included in the analysis of body weights. Differences in the number of piglet deaths in each treatment in each of the 3 weeks were analyzed using a Fisher Exact test.

\section{Results}

\subsection{Experiment 1}

For litters whose teeth were either fully or partially clipped, lesion scores were low, and there were no significant differences between the sides facing the fully clipped or partially clipped teeth (Fig. 1). No litters from this treatment group were removed because of high lesion scores.

For litters where some teeth remained intact, lesion scores on the side facing the intact teeth were significantly greater $(P<0.02)$ than scores on the side facing either fully or partially clipped teeth. Nine litters were removed from the experiment in these two groups, in every case because of high lesion scores on the side facing intact teeth.

\subsection{Experiment 2}

At birth, piglets assigned to the intact treatment weighed (by chance) about $25 \mathrm{~g}$ less than those assigned to the partially and fully clipped treatments (Table 1; N.S.). Despite their slightly lower birth weights, piglets with intact teeth tended to gain more weight during days $0-7$. Weight gain during that first week was positively related to the extent that the teeth were left intact; piglets with fully clipped teeth gained the least, those with partially clipped teeth were intermediate, and those with intact teeth gained the most 
(linear effect of treatment: $P<0.02$; additional nonlinear component: $P>0.2$ ). Weight gains during weeks 2 and 3 were not significantly related to the clipping treatment.

The teat pair occupied by piglets did not vary with treatment. Piglet mortality was low over the course of the experiment (7\%). Deaths were slightly more common among those with intact teeth, but this tendency was not statistically significant.

Fig. 1. Mean facial lesion scores on piglets from the three treatment groups in Experiment 1: (A) intact vs. fully clipped (10 litters); (B) intact vs. partially clipped (9 litters); and (C) partially clipped vs. fully clipped (9 litters). Scores are from the side facing littermates' teeth clipped as indicated. For each of the three panels, the standard error of the difference between the means is illustrated.
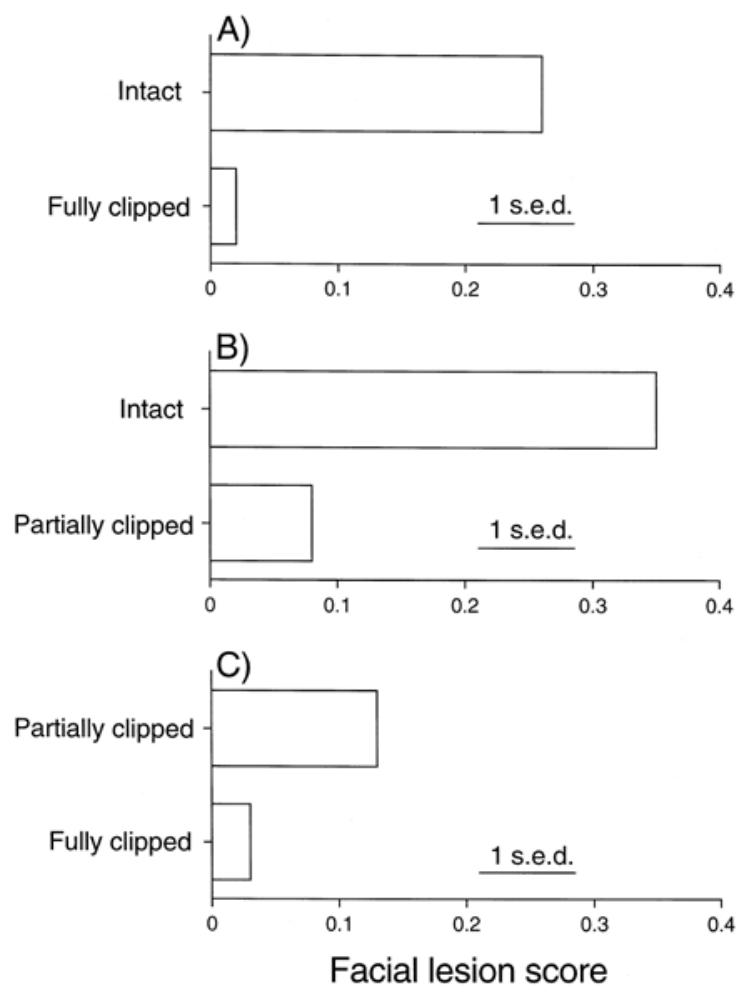

Table 1. Mean (+ s.e.m.) birth weight, weight gains and teat number (1-7, anterior to posterior) as well as number of piglet deaths, for piglets with teeth that were intact, partially clipped, or fully clipped in Experiment 2

\begin{tabular}{|lccc|}
\hline & Intact & Partially clipped & Fully clipped \\
\hline Birth weight (g) & $1268(23)$ & $1285(24)$ & $1294(24)$ \\
Weight gain (g/day) & & & \\
day 0-7 & $154(7)$ & $144(7)$ & $132(6)$ \\
day 7-14 & $201(9)$ & $186(9)$ & $190(9)$ \\
day 14-21 & $206(11)$ & $205(11)$ & $201(10)$ \\
Teat number & $3.47(0.23)$ & $3.47(0.23)$ & $3.23(0.22)$ \\
Piglet deaths & & & 2 \\
day 0-7 & 6 & 2 & 2 \\
day 7-14 & 1 & 2 & 0 \\
day 14-21 & 2 & 1 & 81 \\
$n$ (piglets) & 82 & 78 & \\
\hline
\end{tabular}




\section{Discussion}

Consistent with previous studies, intact teeth caused appreciable facial lesions (e.g., Fraser, 1975; Brown et al., 1996). Experiment 1 demonstrated that teeth with the distal third removed caused minimal lesions — not significantly more than fully clipped teeth and much less than intact teeth.

Experiment 2 showed that piglets with partially clipped teeth were intermediate in weight gain between litter-mates with fully clipped teeth and those with the teeth left intact. The finding of higher gains by piglets with intact teeth (relative to fully clipped litter-mates) is consistent with earlier work (Fraser and Thompson, 1991). It seems likely that retaining intact or partially clipped teeth provided piglets with a competitive advantage, allowing them to achieve slightly higher gains, presumably at the expense of littermates with clipped teeth (Robert et al., 1995). We found no evidence that the competitive advantage involved piglets securing the anterior teats, which tend to be slightly more productive (Fraser and Thompson, 1986). Thus the greater weight gains of piglets with partially clipped and intact teeth may simply be due to these animals maintaining better access to a functional teat (De Passillé and Rushen, 1989). It is also possible that pain or injury to the piglet that resulted from clipping may have affected the piglets' weight gains.

In this study, we did not measure damage to teeth and gums associated with the different clipping treatments. Hutter et al. (1994) found a higher incidence of tooth and gum problems among piglets with clipped teeth than among those where the tips of the teeth had been removed using a grinding tool. Burger (1983) noted that splintering and sharp edges (which are likely to damage the mucous membrane of the lips) were more common when teeth were clipped close to the gum. Thus clipping only the distal third may provide an advantage over clipping to the gum line.

If longer teeth provide piglets with a competitive advantage over their litter-mates, then in herds with intact teeth, genetic selection for rapid gains among piglets may have resulted in inadvertent selection for increased size of the needle teeth. During this study, we noted much variation both between and within litters in the size of the needle teeth before clipping. This raises the possibility that genetic selection for reduced tooth size could make clipping teeth unnecessary.

\section{Acknowledgements}

The experimental work described here was performed at the Centre for Food and Animal Research, prior to its closure. We thank the director and staff for their help with this and other experiments and in maintaining an excellent environment for research. We are especially grateful to Sue Leffler and Lori Bierbrier for their careful work in running Experiments 1 and 2, respectively, to Brian Thompson for his help with the statistical analysis for Experiment 2, and to Ed Pajor for his help and insights in planning both experiments.

\section{References}

Brown, J.M.E., Edwards, S.A., Smith, W.J., Thompson, E., Duncan, J., 1996. Welfare and production implications of teeth clipping and iron injection of piglets in outdoor systems in Scotland. Prev. Vet. Med. 27, 95-105.

Burger, A., 1983. Untersuchungen über die Folgen der Zahnresektion beim Ferkel. Inaugural Dissertation, Tierärztlichen Fakultät der Ludwig-Maximilians-Universität, Munich.

Connor, M.L., 1993. Recommended Code of Practice for Care and Handling of Pigs. Agriculture Canada, Ottawa. 
De Passillé, A.M.B., Rushen, J., 1989. Suckling and teat disputes by neonatal piglets. Appl. Anim. Behav. Sci. 22, 23-38.

Fraser, D., 1975. The 'teat order' of suckling pigs: II. Fighting during suckling and the effects of clipping the eye teeth. J. Agric. Sci. Camb. 84, 393-399.

Fraser, D., Thompson, B.K., 1986. Variation in piglet weights: relationship to suckling behavior, parity number and farrowing crate design. Can. J. Anim. Sci. 66, 31-46.

Fraser, D., Thompson, B.K., 1991. Armed sibling rivalry among suckling piglets. Behav. Ecol. Sociobiol. 29, 9-15.

Hutter, St., Heinritzi, K., Reich, E., Ehret, W., 1994. Efficacité de différentes méthodes de résection des dents chez le porcelet non sevré. Revue Méd. Vét. 145, 205-213.

Robert, S., Thompson, B.K., Fraser, D., 1995. Selective tooth clipping in the management of low-birthweight piglets. Can. J. Anim. Sci. 75, 285-289. 